\title{
PRODUCCIÓN DE FORRAJE Y CALIDAD NUTRICIONAL DE VARIEDADES DE Pennisetum purpureum EN LA MESETA CENTRAL DE COSTA RICA 1
}

\author{
Maritza Araya Mora², Carlos Boschini Figueroa ${ }^{2}$
}

\begin{abstract}
RESUMEN
Producción de forraje y calidad nutricional de variedades de Pennisetum purpureum en la Meseta Central de Costa Rica. El experimento se llevó a cabo en la Estación Experimental Alfredo Volio Mata de la Universidad de Costa Rica, ubicada a $1.542 \mathrm{msnm}$, y con una precipitación media de $2050 \mathrm{~mm}$ al año. Se comparó la producción forrajera, el contenido de materia seca, proteína cruda y cenizas de los pastos Taiwán, King Grass, Gigante, Elefante enano y Camerún establecidos en iguales condiciones y evaluados a diferentes edades de corta. Se determinó que la producción de forraje verde y material seco (kg MS/ha) del pasto King Grass y Taiwán obtuvieron una mayor producción de forraje (15.3 y 13,9 t/ha, respectivamente) seguidos por el Gigante, Camerún y Enano (12,2, 6,9 y 4,7 t/ha, respectivamente). La edad óptima de cosecha para los Pennisetum purpureum evaluados excepto para el Elefante enano fue a los 70 días después del corte, esto debido a la producción y estado fisiológico de las plantas. Para el Elefante enano la cosecha se dio a los 98 y 112 días después del corte. La relación hoja:tallo siempre fue menor a uno, a excepción del Elefante enano con un valor promedio de 1,73. El porcentaje de materia seca aumentó conforme la edad y los mayores contenidos se reportan en las hojas. El contenido de proteína disminuyó al aumentar la edad de corte, al igual que el porcentaje de cenizas, ambos presentaron una mayor cantidad en hojas que en tallos. Se destaca el contenido de proteína del Elefante enano de $16,51 \%$ en planta entera debido a la gran producción de hojas.
\end{abstract}

\begin{abstract}
Nutritional content and forage yield of Pennisetum purpureum varieties in Costa Rican Central Plateau. The experiment was conducted at the Alfredo Volio Mata Experimental Station belongs to the University of Costa Rica, located at an altitude of $1542 \mathrm{~m}$, with annual rainfall of 2,050 $\mathrm{mm}$. Biomass production was compared among Taiwan Grass, King Grass, Elephant, Dwarf Elephant and Cameroon at two different cut ages. It was determined that King Grass and Taiwan produced the largest amounts of green and dry matter (15.3 and $13.9 \mathrm{t} / \mathrm{ha}$, respectively) followed by Elephant, Cameroon and Dwarf Elephant (12.2, 6.9 and 4.7 $\mathrm{t} / \mathrm{ha}$, respectively). It was determined that the greatest production was obtained at 70 days of age due to production and physiological state of the plants. For Dwarf Elephant the optimal age was 98 to 112 days. The shaft:leaf relationship of the evaluated forages were always smaller than one, been the exception the Dwarf Elephant (1.73). Dry matter content increased with age, and the higher content was found in the leaves. Protein levels diminished with cutting age and showed higher levels in leaves than in shafts. Protein content of the Dwarf Elephant was $16.51 \%$ in the whole plant due to the greater production of leaves.
\end{abstract}

Key words: forage, nutritional content, Pennisetum purpureum.

Palabras claves: forraje de corta, calidad nutricional, Pennisetum purpureum, biomasa.

\footnotetext{
1 Recibido: 20 de setiembre, 2004. Aceptado: 17 de diciembre, 2004. Inscrito en Vicerrectoría de Investigación. Proyecto Nº 737-97-006. Universidad de Costa Rica.

2 Estación Experimental Alfredo Volio Mata, Facultad de Ciencias Agroalimentarias, Universidad de Costa Rica. Correo electrónico: marayam@cariari.ucr.ac.cr, boschini@cariari.ucr.ac.cr
} 


\section{INTRODUCCIÓN}

Debido a la competitiva producción que hoy día existe en el sector agropecuario los productores se encuentran obligados a realizar un uso más eficiente de los recursos que poseen. Esto representa entre otras acciones el intensificar la producción por unidad de área. Esta preocupación se ha reflejado en una constante búsqueda de materiales forrajeros que satisfagan los requerimientos nutricionales de sus animales, y a la vez establecer un sistema de cosecha uniforme asegurando un nivel de producción constante durante todo el año.

Existen varios factores internos y externos que limitan la producción de un material forrajero, entre los factores externos más importantes se encuentra el clima. Los países tropicales poseen una diferencia productiva forrajera en la estación seca con respecto a la estación lluviosa, y esto crea una necesidad de alimento en una forma estacional. Otros factores externos son el manejo y las condiciones físicas, químicas y características ecológicas de las tierras en uso, como pendientes altas, mal drenaje, poca fertilidad y baja adaptación de las especies forrajeras.

Dentro los factores internos se encuentra el tipo de material y las etapas fisiológicas de las plantas. El tipo de crecimiento de la planta determina la utilización que se puede hacer, es decir, si se debe destinar para corte o bien para pastoreo y cómo se debe hacer. Las etapas fisiológicas determinan la cantidad de carbohidratos no estructurales como reserva energética para el crecimiento y el área foliar, la interacción entre éstas y el origen del nuevo crecimiento (Bernal 1991).

El uso racional de especies forrajeras de corte y que producen cantidades voluminosas de forraje son una práctica muy común en las actividades pecuarias especialmente las lecheras (Rosa y Silva 1997). Entre esas especies se encuentran los Pennisetum purpureum, su origen se encuentra en África del Sur son robustos, vigorosos y perennes, por lo que han sido introducidos en todas las regiones tropicales y subtropicales (Bernal 1991; Burger 1980). Recientemente, en el país se ha popularizado el uso de un cultivar del Pennisetum purpureum llamado Camerún, este material se caracteriza por poseer un color morado tanto en hojas como en los tallos.

Varias fuentes, en su mayoría no oficiales, para el pasto Camerún en Costa Rica reportan rendimientos en producción de forraje por hectárea muy superiores y una calidad mayor a los encontrados en los Pennisetum purpureum ya conocidos, entre ellos el King Grass, Gigante y Taiwán. Estos datos comparan materiales en diferentes etapas fisiológicas, en donde el Camerún es el forraje más joven analizado. Bernal (1991), indica que las gramíneas que crecen erectas y en cepas dependen casi completamente de sus reservas para el rebrote, ya que la mayor parte del área foliar es removida para su aprovechamiento. A la vez, indica que las cantidades de reservas (carbohidratos no estructurales) fluctúan de acuerdo con la etapa fisiológica del forraje, debido a esto se obtienen rendimientos mayores en materiales más jóvenes a los obtenidos en plantaciones establecidas hace ya varios años.

Los pastos de la familia Pennisetum purpureum son de uso generalizado en los sistemas tropicales, pero no existe una caracterización productiva que defina la producción promedio de los cultivares específicos, por eso es de gran importancia conocer tales fuentes forrajeras, describir su comportamiento fisiológico, la producción y la composición nutricional.

Este trabajo tuvo como objetivo caracterizar y comparar la producción de materia verde y materia seca, así como el contenido materia seca, proteína y cenizas de cinco variedades de Pennisetum purpureum utilizados en Costa Rica, en iguales condiciones.

\section{MATERIALES Y MÉTODOS}

El experimento se llevó a cabo en la Estación Experimental de Ganado Lechero Alfredo Volio Mata de la Universidad de Costa Rica, ubicada en Ochomogo, del Cantón de la Unión en la provincia de Cartago; a $1.542 \mathrm{msnm}$ y con una precipitación media anual de $2050 \mathrm{~mm}$, distribuidos en la época lluviosa que va de mayo a noviembre, posee una humedad relativa media del $84 \%$ y, una temperatura que va de $\operatorname{los} 23^{\circ} \mathrm{C}$ a los 13 ${ }^{\circ} \mathrm{C}$ alcanzando una temperatura media anual de $19,3{ }^{\circ} \mathrm{C}$. El suelo es de origen volcánico, clasificado como Typic Distrandepts, caracterizado por poseer buen drenaje y una fertilidad media (Vásquez 1982).

El experimento se inició en el mes de mayo del 2003 con la preparación y siembra de las cinco variedades (Taiwán, King Grass, Gigante o Elefante, Elefante Enano y Camerún), el área total utilizada fue de 5.272,5 $\mathrm{m}^{2}$, dividida en cinco parcelas similares para cada uno de los pastos. Este terreno permaneció por mucho tiempo en forma de charral, fue necesaria una limpieza del terreno previa a la siembra; posteriormente, se procedió a arar, rastrear y por último surcar. Los surcos se formaron a una distancia de $70 \mathrm{~cm}$ entre ellos. Las cinco variedades de Pennisetum purpureum se sembraron en ese mismo mes.

La siembra dio su inicio con la adición del fertilizante al fondo del surco, aplicando el equivalente de 60 
$\mathrm{kg}$ de fósforo y $12 \mathrm{~kg}$ de nitrógeno por hectárea. Luego, se procedió a colocar la semilla vegetativa en hilera, los tallos se colocaron en forma manual con un traslape de $10 \mathrm{~cm}$ en las puntas. Esta forma de siembra asegura un brote uniforme a lo largo de los surcos. Al estar el material vegetativo colocado se procedió a tapar, con la tierra de los entresurcos. La fertilización y la siembra se realizaron en forma manual.

Ocho semanas después de la siembra se realizó una aplicación a los cultivares con el fertilizante foliar quelatado Bayfoland Forte ${ }^{\circledR}$, para proveer a las plantas de los elementos menores, 10 semanas después de la siembra se aplicó el equivalente de $100 \mathrm{~kg}$ de nitrógeno por hectárea.

El cultivo se desarrolló durante cuatro meses en donde el brote de yemas y crecimiento de los pastos fue muy uniforme. A las cinco semanas de establecido se realizó la aplicación de un herbicida cuyo ingrediente activo es 2,4D para el control de hoja ancha y a las nueve semanas de establecido se utilizó el Piretroide Permethrin, como insecticida para eliminar los insectos chupadores presentes en las parcelas.

$\mathrm{Al}$ cabo de los cuatro meses desde la siembra se procedió a realizar un corte de uniformización y se fertilizó con $150 \mathrm{~kg}$ de nitrógeno por ha en forma de nitrato de amonio, el mismo día en que se realizaron los cortes.

En cada parcela se realizaron cuatro muestreos en los rebrotes a los 70, 84, 98, 112, 126 y 140 días, después del corte, efectuados al azar sin repetir el lugar de muestreo, tomando una muestra de cinco metros lineales dentro del mismo surco a partir del punto escogido al azar por medio del conteo de surcos y distancias dentro de las parcelas. En el Elefante Enano el muestreo se realizó en forma idéntica con un tamaño de muestra de ocho metros lineales. Todo el material cosechado fue pesado en una balanza con una precisión de $\pm 5 \mathrm{~g}$. Todas las muestras de cada edad de corte se realizaron en cuatro repeticiones, el material cosechado se separó en hojas y tallos y se obtuvo el peso correspondiente. Simultáneamente se contó el número de cepas en cada una de las unidades de muestreo. En cada una de las muestras separadas se extrajo una submuestra por repetición para realizar los análisis de contenido de materia seca en una estufa a $105^{\circ} \mathrm{C}$, el contenido proteína cruda por medio de la técnica de Kjendall y el porcentaje de cenizas (AOAC 1990).

Los rendimientos de biomasa muestreados se analizaron con el Proc GLM del paquete estadístico SAS (1985), se efectuó la separación de medias con la prueba de Duncan, en aquellas variables que resultaron significativas (Steel y Torrie 1997).

\section{RESULTADOS Y DISCUSIÓN}

\section{Rendimiento de materia verde y seca}

La producción de biomasa verde promedio medida en tallos, hojas y planta entera $(\mathrm{kg} / \mathrm{ha})$ se muestran en el Cuadro 1. La producción promedio por corte de materia verde en hojas entre los diferentes forrajes evaluados fue significativo $(\mathrm{P} \leq 0,05)$, éstos pueden diferenciarse en tres grupos. El primer grupo lo constituyen el pasto King Grass y Taiwán, los cuales presentaron una producción similar de materia verde en hoja $(24,4$

Cuadro 1. Producción de materia verde, materia seca, proteína cruda y cenizas en hojas, tallos y planta entera en cinco ecotipos de Pennisetum purpureum. Estación Experimental Alfredo Volio Mata, Tres Ríos, Cartago, Costa Rica. 2003.

\begin{tabular}{lcccc}
\hline \multicolumn{1}{c}{ Ecotipo } & Hoja & Tallo & $\begin{array}{c}\text { Planta } \\
\text { entera }\end{array}$ & $\begin{array}{c}\text { Producción } \\
\text { Kg MV/cepa }\end{array}$ \\
& & & & \\
\hline \multicolumn{2}{l}{ Producción de materia verde $(\mathbf{k g} / \mathbf{h a})$} & & \\
Taiwán & $23.893 \mathrm{a}$ & $56.655 \mathrm{a}$ & $80.548 \mathrm{a}$ & $2,03 \mathrm{~b}$ \\
King Grass & $24.431 \mathrm{a}$ & $62.507 \mathrm{a}$ & $86.938 \mathrm{a}$ & $4,37 \mathrm{a}$ \\
Gigante & $18.750 \mathrm{~b}$ & $42.682 \mathrm{~b}$ & $61.432 \mathrm{~b}$ & $1,49 \mathrm{c}$ \\
Enano & $13.401 \mathrm{c}$ & $8.967 \mathrm{~d}$ & $22.368 \mathrm{~d}$ & $0,61 \mathrm{~d}$ \\
Camerún & $12.100 \mathrm{c}$ & $23,57 \mathrm{c}$ & $35.457 \mathrm{c}$ & $0,95 \mathrm{~d}$ \\
\hline Producción de materia seca (kg/ha) & & Relación \\
& & & & hoja:tallo \\
& & & & \\
Taiwán & $5.048 \mathrm{a}$ & $8.818 \mathrm{a}$ & $13.866 \mathrm{a}$ & $0,65 \mathrm{~b}$ \\
King Grass & $5.094 \mathrm{a}$ & $10.168 \mathrm{a}$ & $15.262 \mathrm{a}$ & $0,62 \mathrm{bc}$ \\
Gigante & $4.005 \mathrm{~b}$ & $8.239 \mathrm{~b}$ & $12.244 \mathrm{~b}$ & $0,54 \mathrm{c}$ \\
Enano & $2.873 \mathrm{c}$ & $1.784 \mathrm{~d}$ & $4.658 \mathrm{~d}$ & $1,73 \mathrm{a}$ \\
Camerún & $2.462 \mathrm{c}$ & $4.471 \mathrm{c}$ & $6.934 \mathrm{c}$ & $0,65 \mathrm{~b}$ \\
\hline
\end{tabular}

$\begin{array}{lcrr}\text { Contenido de materia seca }(\boldsymbol{\%}) & \\ \text { Taiwán } & 20,49 \mathrm{a} & 18,20 \mathrm{~b} & 19,44 \mathrm{~b} \\ \text { King Grass } & 21,40 \mathrm{a} & 19,95 \mathrm{a} & 21,04 \mathrm{a} \\ \text { Gigante } & 21,51 \mathrm{a} & 18,68 \mathrm{~b} & 19,82 \mathrm{~b} \\ \text { Enano } & 21,03 \mathrm{a} & 15,38 \mathrm{c} & 17,80 \mathrm{c} \\ \text { Camerún } & 21,08 \mathrm{a} & 14,71 \mathrm{c} & 17,17 \mathrm{c}\end{array}$

Contenido de proteína cruda $(\%)$

$\begin{array}{llll}\text { Taiwán } & 13,69 \mathrm{~b} & 7,29 \mathrm{~b} & 9,73 \mathrm{~b}\end{array}$

King Grass $\quad 13,18$ bc $\quad 6,67 \mathrm{c} \quad 9,08 \mathrm{~d}$

Gigante $\quad 14,89$ a $\quad 6,29 \mathrm{~d} \quad 9,30 \mathrm{~cd}$

Enano $\quad 12,72 \mathrm{c} \quad 8,5 \mathrm{a} \quad 11,14 \mathrm{a}$

Camerún $\quad 13,69 \mathrm{~b} \quad 7,08 \mathrm{~b} \quad 9,65 \mathrm{~b} \mathrm{c}$

Contenido de cenizas (\%)

Taiwán $\quad 22,07 \mathrm{~b} \quad 13,83 \mathrm{~b} \quad 16,89 \mathrm{~b}$

King Grass $\quad 20,93$ c $\quad 12,64$ c $\quad 15,54$ c

$\begin{array}{llll}\text { Gigante } & 19,52 \mathrm{~d} & 12,90 \mathrm{c} & 15,10 \mathrm{c}\end{array}$

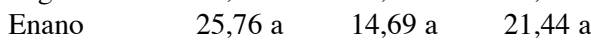

Camerún $\quad 21,73 \mathrm{~b} \quad 13,81 \mathrm{~b} \quad 16,81 \mathrm{~b}$

a, b, c, d muestran diferencias significativas $(P \leq 0,05)$. 
y 23,9t/ha, respectivamente), luego el pasto Gigante con un rendimiento menor, y por últimos el Elefante Enano y Camerún, éste último presentó una producción de hoja menor que el Elefante Enano; sin embargo, no se presentaron diferencias significativas $(P \geq 0,05)$ entre ellos. En el Cuadro 1 se muestra que el pasto Camerún presentó una producción baja de forraje verde por cepa $(0,96 \mathrm{~kg})$, con respecto al King Grass y Taiwán $(4,37$ y $2,03 \mathrm{~kg}$, respectivamente). La producción de material verde en tallos mostró un comportamiento semejante a la hoja, excepto en el pasto Elefante Enano, debido a las características de porte más bajo y menor producción de tallo, posee el menor rendimiento respecto a los demás forrajes evaluados. La producción de materia verde total de los pastos Pennisetum purpureum muestran una tendencia de producción similar a los rendimientos de materia verde de los tallos.

La relación hoja: tallo en base seca de los pastos evaluados fue inferior a uno de los cultivares de Pennisetum purpureum estudiados, excepto en el Pasto Elefante enano o Mott, que reporta un valor de 1,73 (Cuadro 1). Aragón (1996), encontró un promedio en la relación hoja:tallo de 1,3 en el pasto Elefante Enano bajo diferentes intensidades de pastoreo.

Los rendimientos de materia seca por corte en tallos, hojas y planta entera se observan en el Cuadro 1, el valor mayor en toneladas por hectárea lo obtuvo el King Grass seguido por el Taiwán (15,2 y 13,8, respectivamente), y por el pasto Gigante $(12,2)$. El Elefante Enano obtuvo el rendimiento menor con 4,7 t/ha presentando diferencias significativas $(\mathrm{P} \leq 0,05)$ con respecto al pasto Camerún con 6,9 t/ha de material seco.

\section{Calidad nutricional de los cultivares evaluados}

Los contenidos de materia seca en hoja, tallo y planta entera se presentan en el Cuadro 1. Los contenidos mayores siempre se presentaron en las hojas de los materiales evaluados. Los valores de materia seca en hojas de los cultivares evaluados no presentaron diferencias ente ellos $(\mathrm{P} \geq 0,05)$. El Elefante Enano tuvo la mayor concentración de materia seca en tallo $(\mathrm{P} \leq 0,05)$, luego el Gigante y Camerún, sin diferencias significativas entre ellos $(\mathrm{P} \geq 0,05)$, con los menores contenidos de materia seca en el tallo se encuentran el King Grass y Taiwán, tales diferencias pueden asociarse al grosor de los tallos, debido a que entre más pequeños y delgados, éstos poseen una mayor cantidad de materia seca. Los contenidos de materia seca en la planta entera mostraron la misma tendencia al contenido en los tallos.

El contenido proteico de los cinco cultivares de Pennisetum purpureum en hojas, tallo y planta entera se muestran en el Cuadro 1. La proteína cruda presente en las hojas varió entre 12,72 y 14,89\%, que corresponden al Elefante Enano y Gigante, respectivamente. Los valores intermedios se presentaron en el Camerún, King Grass y Taiwán, en orden descendente. El Elefante enano mostró el mayor contenido medio de proteína cruda en tallos $(8,5 \%)(\mathrm{P} \leq 0,05)$, seguido del Taiwán, Camerún, King Grass y Gigante (7,29; 7,08; 6,67 y 6,29\%, respectivamente). El pasto Elefante Enano mostró el mayor contenido de proteína cruda presente en la planta entera con respecto a los demás materiales evaluados $(\mathrm{P} \leq 0,05)$, debido a la alta producción de hoja el cual le confiere una mejor calidad nutricional que los demás Pennisetum evaluados. Al respecto Rodríguez y Capo De Blanco (1971) citados por Carmona y Rodríguez, estudiaron la composición química y determinaron la materia seca en los tallos y hojas de 21 cultivares de Pasto Elefante y concluyeron que los cultivares con mayor proporción de hojas tienen un mayor valor nutritivo.

El contenido de cenizas se presenta en el Cuadro 1. Los valores de ceniza que se encontraron en las hojas fueron más elevados que en tallos. El pasto Elefante enano presentó el mayor contenido de esta fracción $(\mathrm{P} \leq 0,05)$ en hojas, tallos y por consiguiente en la planta entera.

\section{Comportamiento productivo de los cultivares Pen- nisetum $p$. evaluados a diferentes edades}

Los cultivares Pennisetum purpureum en general presentaron una mayor producción de hojas y tallos conforme avanzó la edad de ellos. Este crecimiento no fue proporcional debido a que la producción de material en forma de tallo superó a la producción de hoja, obteniendo entonces una relación hoja:tallo menor conforme avanza la edad de corte en los cinco pastos evaluados (Cuadro 2).

El contenido de materia seca en hoja, tallo y planta entera fue mayor conforme la edad de los pastos aumentó. A excepción de los 140 días, donde el porcentaje de materia seca en hoja disminuyó en un $9 \%$ con respeto a los 126 días del corte. El contenido de proteína cruda también disminuyó conforme la edad de corte avanzó, fue mayor a los 70 días y menor a los 140 días del corte. El contenido de cenizas en hojas se mantuvo constante conforme aumentó la edad de los pastos. En tallos y planta entera el contenido de cenizas diminuyó conforme aumentó la edad de corte (Cuadro 2).

El comportamiento productivo a través del tiempo, de cada uno de los Pennisetum purpureum evaluados, corresponden al estado fenológico del forraje, los cuales presentaron diferencias entre sí (Cuadro 3). Los 
Cuadro 2. Producción de materia verde, materia seca, proteína cruda y cenizas en hojas, tallos y planta entera de los pastos Pennisetum purpureum a diferentes edades de corte. Estación Experimental Alfredo Volio Mata, Tres Ríos, Cartago. 2003.

\begin{tabular}{ccccc}
\hline Edad & Hoja & Tallo & $\begin{array}{c}\text { Planta } \\
\text { entera }\end{array}$ & $\begin{array}{c}\text { Producción } \\
\text { kg MV/cepa }\end{array}$ \\
\hline \multicolumn{5}{c}{ Producción de materia verde (kg/ha) } \\
70 & $18.989 \mathrm{a}$ & $32.389 \mathrm{c}$ & $51.377 \mathrm{~b}$ & $1,76 \mathrm{~b}$ \\
84 & $17.274 \mathrm{a}$ & $32.189 \mathrm{c}$ & $49.462 \mathrm{~b}$ & $1,91 \mathrm{~b}$ \\
98 & $17.873 \mathrm{a}$ & $34.538 \mathrm{c}$ & $52.411 \mathrm{~b}$ & $1,23 \mathrm{c}$ \\
112 & $20.526 \mathrm{a}$ & $44.694 \mathrm{ab}$ & $65.219 \mathrm{a}$ & $1,95 \mathrm{~b}$ \\
126 & $17.559 \mathrm{a}$ & $39.887 \mathrm{bc}$ & $57.446 \mathrm{ab}$ & $1,85 \mathrm{~b}$ \\
140 & $18.870 \mathrm{a}$ & $49.304 \mathrm{a}$ & $68.174 \mathrm{a}$ & $2,66 \mathrm{a}$ \\
\hline \multicolumn{7}{c}{} \\
Producción de materia seca (kg/ha) & & Relación \\
70 & $2.744 \mathrm{c}$ & $2.928 \mathrm{~d}$ & $5.672 \mathrm{~d}$ & $1,14 \mathrm{a}$ \\
84 & $3.022 \mathrm{~b}$ & $3.858 \mathrm{c}$ & $6.880 \mathrm{~cd}$ & $0,98 \mathrm{~b}$ \\
98 & $3.643 \mathrm{~b}$ & $4.945 \mathrm{c}$ & $8.588 \mathrm{c}$ & $0,91 \mathrm{~b}$ \\
112 & $4.995 \mathrm{a}$ & $7.982 \mathrm{~b}$ & $12.977 \mathrm{~b}$ & $0,80 \mathrm{c}$ \\
126 & $4.570 \mathrm{a}$ & $8.262 \mathrm{~b}$ & $12.831 \mathrm{~b}$ & $0,66 \mathrm{~d}$ \\
140 & $4.406 \mathrm{a}$ & $12.202 \mathrm{a}$ & $16.608 \mathrm{a}$ & $0,54 \mathrm{e}$ \\
\hline
\end{tabular}

Contenido de materia seca (\%)

\begin{tabular}{rrrr}
70 & $14,71 \mathrm{e}$ & $10,13 \mathrm{f}$ & $12,40 \mathrm{f}$ \\
84 & $17,82 \mathrm{~d}$ & $13,21 \mathrm{e}$ & $15,30 \mathrm{e}$ \\
98 & $21,01 \mathrm{c}$ & $15,06 \mathrm{~d}$ & $17,72 \mathrm{~d}$ \\
112 & $24,00 \mathrm{~b}$ & $18,07 \mathrm{c}$ & $20,42 \mathrm{c}$ \\
126 & $25,69 \mathrm{a}$ & $21,83 \mathrm{~b}$ & $23,15 \mathrm{~b}$ \\
140 & $23,38 \mathrm{~b}$ & $26,00 \mathrm{a}$ & $25,33^{\mathrm{a}}$ \\
\hline
\end{tabular}

$\begin{array}{cccc}\text { Contenido de proteína cruda }(\%) \\ 70 & 16,56 \mathrm{a} & 10,53 \mathrm{a} & 13,51 \mathrm{a} \\ 84 & 15,91 \mathrm{a} & 8,69 \mathrm{~b} & 11,90 \mathrm{~b} \\ 98 & 14,18 \mathrm{~b} & 7,63 \mathrm{c} & 10,35 \mathrm{c} \\ 112 & 12,89 \mathrm{c} & 6,24 \mathrm{~d} & 8,90 \mathrm{~d} \\ 126 & 11,75 \mathrm{~d} & 5,23 \mathrm{e} & 7,63 \mathrm{e} \\ 140 & 10,51 \mathrm{e} & 4,68 \mathrm{f} & 6,36 \mathrm{f}\end{array}$

\begin{tabular}{clll}
\multicolumn{5}{c}{ Contenido de cenizas $(\%)$} \\
70 & $21,72 \mathrm{bc}$ & $16,79 \mathrm{a}$ & $19,38, \mathrm{a}$ \\
84 & $22,321 \mathrm{ab}$ & $14,97 \mathrm{~b}$ & $18,59 \mathrm{~b}$ \\
98 & $22,79 \mathrm{a}$ & $14,90 \mathrm{~b}$ & $18,46 \mathrm{~b}$ \\
112 & $21,18 \mathrm{c}$ & $12,82 \mathrm{c}$ & $16,47 \mathrm{c}$ \\
126 & $21,56 \mathrm{bc}$ & $11,02 \mathrm{~d}$ & $15,18 \mathrm{~d}$ \\
140 & $22,43 \mathrm{ab}$ & $10,95 \mathrm{~d}$ & $14,85 \mathrm{~d}$
\end{tabular}

a, b, c, d, e muestran diferencias significativas $(P \leq 0,05)$.

pastos King Grass, Gigante y Taiwán tuvieron un comportamiento cíclico de aumento en $\mathrm{kg} / \mathrm{ha} /$ corte de materia seca en hojas a los 84,112 y 140 , excepto para el King Grass que presentó una disminución a los 140 días. La disminución en $\mathrm{kg} / \mathrm{ha} /$ corte de materia seca para los tres pastos citados se manifestó a los 98 y 126 días. En tallos el comportamiento fue similar al de
Cuadro 3. Producción de materia seca en cinco ecotipos de Pennisetum purpureum a diferentes edades de corte. Estación Experimental Alfredo Volio Mata, Tres Ríos, Cartago. 2003.

\begin{tabular}{|c|c|c|c|c|c|}
\hline \multirow{2}{*}{$\begin{array}{c}\text { Tipo } \\
\text { Pasto }\end{array}$} & \multirow{2}{*}{$\begin{array}{l}\text { Edad } \\
\text { días }\end{array}$} & \multicolumn{3}{|c|}{ Materia seca (kg/ha/corte) } & \multirow{2}{*}{$\begin{array}{l}\text { Relación } \\
\text { hoja:tallo }\end{array}$} \\
\hline & & Hoja & Tallo & Total & \\
\hline \multirow[t]{6}{*}{ Taiwán } & 70 & 2.811 & 3.134 & 5.945 & 0.89 \\
\hline & 84 & 3.946 & 5.266 & 9.212 & 0.76 \\
\hline & 98 & 4.429 & 6.402 & 10.831 & 0.70 \\
\hline & 112 & 6.199 & 11.358 & 17.557 & 0.56 \\
\hline & 126 & 6.215 & 11.399 & 17.614 & 0.54 \\
\hline & 140 & 6.688 & 15.349 & 22.037 & 0.44 \\
\hline \multirow[t]{6}{*}{ King Grass } & 70 & 3.664 & 3.687 & 7.350 & 1.00 \\
\hline & 84 & 3.793 & 5.255 & 9.048 & 0.71 \\
\hline & 98 & 4.364 & 7.260 & 11.624 & 0.61 \\
\hline & 112 & 7.339 & 13.495 & 20.834 & 0.54 \\
\hline & 126 & 6.238 & 11.388 & 17.626 & 0.56 \\
\hline & 140 & 5.163 & 19.924 & 25.087 & 0.28 \\
\hline \multirow[t]{6}{*}{ Gigante } & 70 & 2.871 & 4.354 & 7.224 & 0.65 \\
\hline & 84 & 3.595 & 5.801 & 9.396 & 0.63 \\
\hline & 98 & 3.338 & 6.015 & 9.353 & 0.57 \\
\hline & 112 & 4.814 & 8.383 & 13.196 & 0.58 \\
\hline & 126 & 4.563 & 9.174 & 13.736 & 0.50 \\
\hline & 140 & 4.849 & 15.709 & 20.559 & 0.33 \\
\hline \multirow[t]{6}{*}{ Enano } & 70 & 1.992 & 909 & 2.901 & 2.21 \\
\hline & 84 & 2.452 & 1.188 & 3.640 & 2.04 \\
\hline & 98 & 4.006 & 2.059 & 6.065 & 2.00 \\
\hline & 112 & 3.490 & 2.204 & 5.695 & 1.62 \\
\hline & 126 & 2.909 & 2.503 & 5.412 & 1.21 \\
\hline & 140 & 2.393 & 1.843 & 4.236 & 1.30 \\
\hline \multirow[t]{6}{*}{ Camerún } & 70 & 2.381 & 2.557 & 4.938 & 0.93 \\
\hline & 84 & 1.324 & 1.780 & 2.104 & 0.78 \\
\hline & 98 & 2.078 & 2.992 & 5.070 & 0.70 \\
\hline & 112 & 3.134 & 4.469 & 7.603 & 0.71 \\
\hline & 126 & 2.923 & 6.844 & 9.767 & 0.46 \\
\hline & 140 & 2.934 & 8.186 & 11.120 & 0.36 \\
\hline
\end{tabular}

hojas. El King Grass presentó un aumento en la producción de tallos a los 140 días del corte debido al comportamiento cíclico de disminución de hojas y aumento en el rebrote de tallos.

El pasto Camerún presentó la máxima producción de materia seca en hoja por hectárea por corte a los 70 y 112 días, y la menor producción a los 84 días, el tallo presentó la menor producción de materia a los 84 días del corte. Esto refleja un ciclo de producción de forraje más temprano que en los demás Pennisetum evaluados (menor a los 70 días). El pasto Elefante Enano presentó la máxima producción de material seco a los 98 días, en tallos la producción aumentó conforme avanzó la edad.

Los contenidos de materia seca, proteína cruda y cenizas por cultivar a través del periodo de crecimiento se presentan en el Cuadro 4 . Todos los forrajes analizados 
Cuadro 4. Contenidos de materia seca, proteína cruda y cenizas de cinco ecotipos de Pennisetum purpureum a diferentes edades de corte. Estación Experimental Alfredo Volio Mata, Tres Ríos, Cartago. 2003.

\begin{tabular}{|c|c|c|c|c|c|c|c|c|c|c|}
\hline \multirow[t]{2}{*}{ Ecotipo } & \multirow{2}{*}{$\begin{array}{l}\text { Edad } \\
\text { (días) }\end{array}$} & \multicolumn{3}{|c|}{ Materia seca $(\%)$} & \multicolumn{3}{|c|}{ Proteína cruda (\%) } & \multicolumn{3}{|c|}{ Cenizas (\%) } \\
\hline & & Hoja & Tallo & $\begin{array}{l}\text { Planta } \\
\text { entera }\end{array}$ & Hoja & Tallo & $\begin{array}{l}\text { Planta } \\
\text { entera }\end{array}$ & Hoja & Tallo & $\begin{array}{l}\text { Planta } \\
\text { entera }\end{array}$ \\
\hline \multirow[t]{6}{*}{ Taiwán } & 70 & 12,47 & 7,55 & 9,87 & 14,51 & 10,80 & 12,52 & 21,66 & 17,62 & 19,53 \\
\hline & 84 & 16,22 & 10,82 & 13,15 & 15,21 & 8,55 & 11,40 & 24,16 & 15,49 & 19,22 \\
\hline & 98 & 18,72 & 12,91 & 15,30 & 14,33 & 7,12 & 10,07 & 23,24 & 16,99 & 19,55 \\
\hline & 112 & 23,79 & 17,28 & 19,65 & 14,31 & 6,23 & 9,10 & 20,78 & 12,91 & 15,74 \\
\hline & 126 & 29,45 & 19,53 & 23,03 & 12,17 & 5,26 & 7,70 & 21,16 & 10,68 & 14,38 \\
\hline & 140 & 25,80 & 20,19 & 22,01 & 11,62 & 5,81 & 7,60 & 21,43 & 9,26 & 12,91 \\
\hline \multirow[t]{6}{*}{ King Grass } & 70 & 13,93 & 7,30 & 10,63 & 14,81 & 9,99 & 12,43 & 21,00 & 16,32 & 18,67 \\
\hline & 84 & 17,27 & 10,44 & 13,28 & 15,26 & 8,14 & 11,10 & 21,03 & 14,33 & 17,11 \\
\hline & 98 & 19,38 & 12,98 & 15,41 & 14,60 & 7,68 & 10,28 & 21,82 & 13,60 & 16,71 \\
\hline & 112 & 26,94 & 17,34 & 20,72 & 12,85 & 5,71 & 8,22 & 19,81 & 11,86 & 14,65 \\
\hline & 126 & 27,69 & 18,50 & 21,83 & 11,10 & 4,53 & 6,88 & 20,75 & 9,92 & 13,83 \\
\hline & 140 & 20,97 & 25,74 & 24,94 & 10,48 & 3,96 & 5,37 & 21,14 & 9,82 & 12,26 \\
\hline \multirow[t]{6}{*}{ Gigante } & 70 & 14,94 & 11,32 & 12,76 & 17,95 & 9,25 & 12,67 & 20,70 & 18,18 & 19,18 \\
\hline & 84 & 17,58 & 13,86 & 15,29 & 17,96 & 7,48 & 11,52 & 20,47 & 14,78 & 16,94 \\
\hline & 98 & 21,67 & 17,25 & 18,85 & 14,91 & 6,24 & 9,34 & 19,22 & 12,59 & 14,97 \\
\hline & 112 & 25,19 & 20,38 & 22,15 & 13,53 & 5,73 & 8,60 & 18,42 & 11,72 & 14,17 \\
\hline & 126 & 25,52 & 21,54 & 22,86 & 14,40 & 5,49 & 8,44 & 18,68 & 9,65 & 12,65 \\
\hline & 140 & 24,18 & 27,75 & 27,04 & 10,58 & 3,57 & 5,23 & 19,61 & 10,50 & 12,69 \\
\hline \multirow[t]{6}{*}{ Enano } & 70 & 16,15 & 13,78 & 15,45 & 18,26 & 12,73 & 16,51 & 23,29 & 16,90 & 21,23 \\
\hline & 84 & 20,11 & 16,91 & 19,07 & 15,26 & 10,64 & 13,71 & 23,33 & 14,80 & 20,51 \\
\hline & 98 & 22,98 & 17,24 & 21,06 & 12,52 & 9,21 & 11,41 & 28,07 & 16,81 & 24,37 \\
\hline & 112 & 22,31 & 19,23 & 21,12 & 11,85 & 7,72 & 10,24 & 25,48 & 14,52 & 21,21 \\
\hline & 126 & 22,61 & 24,82 & 23,66 & 10,04 & 5,55 & 7,98 & 26,39 & 12,60 & 20,08 \\
\hline & 140 & 24,27 & 27,76 & 25,86 & 8,38 & 5,16 & 6,98 & 28,02 & 12,46 & 21,25 \\
\hline \multirow[t]{6}{*}{ Camerún } & 70 & 16,08 & 10,73 & 13,31 & 17,28 & 9,89 & 13,43 & 21,95 & 14,93 & 18,28 \\
\hline & 84 & 17,93 & 14,06 & 15,73 & 15,87 & 8,63 & 11,76 & 22,62 & 15,43 & 18,53 \\
\hline & 98 & 22,31 & 14,95 & 17,97 & 14,56 & 7,91 & 10,64 & 21,60 & 14,44 & 17,37 \\
\hline & 112 & 21,79 & 16,12 & 18,48 & 11,90 & 5,79 & 8,31 & 21,44 & 13,12 & 16,55 \\
\hline & 126 & 23,17 & 24,76 & 24,38 & 11,05 & 5,36 & 7,15 & 20,82 & 12,24 & 14,95 \\
\hline & 140 & 21,71 & 28,57 & 26,79 & 11,48 & 4,88 & 6,62 & 21,95 & 12,71 & 15,15 \\
\hline
\end{tabular}

en la presente investigación obtuvieron los rendimientos mayores de porcentaje de materia seca en planta entera a los 140 días después del corte, con excepción del pasto Taiwán que se presentó a los 126 días, debido al aumento en el porcentaje de materia seca en hojas a esa edad. En general, los porcentajes de proteína cruda de los pastos evaluados decayeron conforme aumenta la edad de corte, debido a la menor presencia de hojas. La alta relación hoja:tallo del Elefante Enano a los 70 días le confirió los mayores contenidos de proteína cruda, los menores contenidos de proteína cruda los presentó el pasto Gigante a los 140 días de corte.

Los mayores contenidos de cenizas se encontraron en las hojas, estos contenidos variaron de $18 \%$ en el pasto Gigante a los 112 días del corte hasta $28 \%$ en el Ele- fante Enano a los 98 días. En tallos, la tendencia de los contenidos de cenizas fue a disminuir conforme aumentó la edad de corte. El comportamiento de las cenizas en planta entera fue descendiente con respecto a la edad de corte para todos los cultivares evaluados excepto para el Elefante Enano. Debido al alto contenido de cenizas en hojas y a la relación hoja:tallo de este pasto, el porcentaje de cenizas fue mayor a los 98 días del corte.

\section{CONCLUSIONES Y RECOMENDACIONES}

Se determinó los rendimientos de material verde y seco que producen los pastos Pennisetum purpureum estudiados. De ellos el King Grass y el Taiwán $(\mathrm{p} \geq 0,05)$ 
tuvieron una producción de biomasa por hectárea superior al reportado por el pasto Camerún $(\mathrm{p} \leq 0,05)$, evaluados en iguales condiciones.

Los pastos Gigante, Camerún y Elefante Enano obtuvieron una producción de forraje verde de hasta un 30,60 , y 75 por ciento menos que el King Grass, respectivamente; sin embargo, cabe destacar que el Elefante Enano a pesar del bajo rendimiento en biomasa posee una composición botánica ideal debido a que la relación hoja:tallo de los forrajes fueron menores a uno con excepción de este material con diferencias significativas ( $\leq \leq 0,05)$, esta característica influye en una buena composición nutricional del Elefante Enano.

En los pastos King Grass, Taiwán, Gigante y Camerún la edad para aprovecharlos es a los 70 días después del corte. Además, alrededor de los 112 y de nuevo a los 140 días se obtuvieron cantidades de biomasa total mayores que los obtenidos a los 70 días después del corte, lo anterior debido al comportamiento del ciclo de crecimiento de las plantas en donde se produce mayor material, sin embargo, la magnitud de la producción por unidad de área que se obtienen a esas edades de corta no justifica el tiempo que se debe esperar para obtenerlos. Además, la producción de tallo aumenta en forma importante manteniendo la cantidad de hojas casi constante, afectando negativamente la relación hoja:tallo de los forrajes evaluados conforme aumenta la edad de éstos (Cuadros 2 y 3).

Para el pasto Pennisetum purpureum cv Mott (Elefante Enano) la fecha óptima de cosecha varió con respecto a los demás forrajes evaluados, para el Elefante Enano se obtuvo la mayor cantidad de hoja entre los 98 y 112 días después del corte, coincidiendo con una mayor producción de materia verde total.

Los contenidos de materia seca aumentaron conforme a la edad de corte. Los contenidos proteína cruda y cenizas disminuyeron conforme avanzó la edad de corte.

La edad de corte ideal para los Pennisetum estudiados varió según su estado fenológico, es decir, los resultados reportados a partir de comparaciones realizadas entre cultivos a diferentes edades y prácticas agronómicas no son consistentes con el potencial real de cada cultivar. Se recomienda realizar investigaciones donde la cosecha se realice en función a la composición fenológica de los cultivos.

\section{LITERATURA CITADA}

ARAGON, C.A. 1996. Productividad y calidad nutritiva del Pasto Elefante Enano (Pennisetum purpureum Schum) en la Zona Atlántica de Costa Rica: Diferentes combinaciones de frecuencias e intensidades de pastoreo. Tesis Presentada para optar por el Título de Ingeniero Agrónomo con énfasis en Zootecnia. Universidad de Costa Rica. San José, Costa Rica. 61 p.

AOAC (Association of official Analitical Chemists). 1990. Official methods of analysis. 15th ed. Arlington, Virginia, USA. 168 p.

BERNAL, J.E. 1991. Pastos y forrajes tropicales. Producción y manejo. Unidad de Divulgación y Prensa. Banco Ganadero. 2a Ed. Bogotá, Colombia. 544 p.

BURGER, W. 1980. Flora costarricensis. Publisher by Fiel Museum of Natural History. 4th Edition. United Estates of America. 608 p.

CARMONA, A; RODRÍGUEZ, H. 1979. Comparación de nueve cultivares y un híbrido de Pasto Elefante (Pennisetum purpureum Schum) en el sur del Lago de Maracaibo. Revista de la Facultad de Agronomía (LUZ) 5(3):514-521.

ROSA, B.; SILVA, S.R.C. 1997. Efeito das épocas de diferimento na produçao e composiçao química do capin-elefante (Pennisetum purpureum, Schum. cv. Cameroon). Anais das Escolas de Agronomia e Veterinaria. Goiânia, Universidade Federal de Goiás. 27(2): 109-115.

SAS (Statistical Analysis System Institute Inc. US). 1985. SAS user's guide: Statistics (Version 5). Cary, NC. 373 p.

STEEL, R.; TORRIE, J. 1997. Bioestadística: Principios y procedimientos. McGraw Hill. México, D.F. 622 p.

VASQUEZ, A. 1982. Estudio detallado de los suelos de la Estación Experimental de Ganado Lechero El Alto. Escuela de Fitotecnia, Facultad de Agronomía, Universidad de Costa Rica. San José, Costa Rica. p. 36. 